\title{
COMPOSITION GRADIENTS IN SPIRAL GALAXIES
}

\author{
MANUEL PEIMBERT \\ Instituto de Astronomia, UNAM \\ Apartado Postal 70-264, México 04510, D. F.
}

\section{Abstract}

Some topics on composition gradients are discussed. One of the most important processes responsible for the abundance gradients is the variation of the low-mass end of the IMF with $\mathrm{O} / \mathrm{H}$.

\section{What causes the $\mathrm{O} / \mathrm{H}$ gradient ?}

There are well established gradients in the Galaxy and other spiral galaxies (e. g. Vila-Costas \& Edmunds, 1992; Henry et al., 1992; Zaritsky, Kennicutt \& Huchra, 1994; Henry \& Howard, 1994; Vila-Costas, this volume). There are at least ten possible explanations that have been proposed in the literature for the presence of abundance gradients: $(i)$ a variation of the Initial Mass Function, IMF, at the low-mass end with $\mathrm{O} / \mathrm{H},(\mathrm{ii})$ a variation of the slope of the IMF at the high-mass end with $\mathrm{O} / \mathrm{H},($ iii) an increase of the oxygen yield, $y(\mathrm{O})$, with $\mathrm{O} / \mathrm{H}$ for a given $\mathrm{IMF},(i v)$ a decrease with $\mathrm{O} / \mathrm{H}$ of the fraction of massive stars that enrich the ISM due to the production of black holes, $(v)$ an increase of $M_{\text {gas }} / M_{\text {tot }}$ with galactocentric distance, $R,(v i)$ an increase of $M_{d a r k} / M_{\text {tot }}$ with $R$, (vii) an increase of the outflow rate over the star formation rate, SFR, with $R$, (viii) an increase of the infall rate over the SFR with $R,(i x)$ the existence of inward flows due to gas with low angular momentum, and $(x)$ the existence of gas flows caused by viscous transfer of angular momentum across the disk.

The observed gradients are $\sim-0.07 \mathrm{dex} \mathrm{kpc}^{-1}$, which implies that if any of the above effects poduces a gradient of this value, then the other nine effects become irrelevant. The first four explanations have to do with stellar properties, $(v)$ and $(v i)$ with galactic chemical evolution under the assumption of a closed model (Searle \& Sargent, 1972), and the last four 
with gas flows. A discussion of some of these effects has been given by Pagel $(1989,1992 \mathrm{a}, 1994)$.

There is growing evidence that the fraction of low-mass stars in the IMF decreases with $\mathrm{O} / \mathrm{H}$. To explain the increase of the observed yield with metallicity, Peimbert \& Serrano (1982) suggested that the fraction of lowmass stars decreases with $\mathrm{O} / \mathrm{H}$, this decrement implies $\Delta y(\mathrm{O})=-0.05 \mathrm{dex}$ $\mathrm{kpc}^{-1}$. Güsten \& Mezger (1982) proposed a bimodal star formation with an increasing fraction of low-mass stars with $R$, which implies $\Delta y(\mathrm{O})=-0.05$ dex $\mathrm{kpc}^{-1}$. Larson (1986) mentions that a model with a constant IMF can be ruled out for the inner galactic disk since it would predict more mass in low-mass stars than is allowed by the rotation curve. Richer et al. (1991) find a steeper slope for the IMF of globular clusters than for the solar vicinity, implying that the fraction of small-mass stars increases with decreasing $\mathrm{O} / \mathrm{H}$. From chemical evolution models of irregular galaxies and of the solar vicinity, Carigi et al. (1994) also find $\Delta y(0)=-0.06 \mathrm{dex}$ $\mathrm{kpc}^{-1}$, mainly due to changes in the fraction of stars at the low-mass end of the IMF.

From the comparison of a grid of ionization structure models with the observations of H II regions in different galaxies, McGaugh (1991) has found that no variation with $\mathrm{O} / \mathrm{H}$ of the slope of the IMF at the high-mass end is present. From the computations by Maeder (1992) for case A, i. e. without black holes, it is found that $y(\mathrm{O})$ decreases with $\mathrm{O} / \mathrm{H}$ producing an increase in $\nabla(\mathrm{O} / \mathrm{H})$ with $R$; alternatively, from cases $\mathrm{B}$ to $\mathrm{E}$, those which include black holes, it is found that $y(\mathrm{O})$ increases with $\mathrm{O} / \mathrm{H}$ producing a decrease in $\nabla(\mathrm{O} / \mathrm{H})$ with $R$. Black holes preventing the production of SN II at the end of the evolution of massive stars, have been ruled out by galactic chemical evolution models (e. g. Carigi, 1994; Peimbert, Sarmiento \& Colín, 1994).

From the variation of $M_{\text {gas }} / M_{\text {tot }}$ in the Galaxy and assuming a closed model for the galactic chemical evolution, Güsten \& Mezger (1982) find that $\nabla(\mathrm{O} / \mathrm{H})=-0.01 \mathrm{dex} \mathrm{kpc}^{-1}$, while Pagel $(1989)$ finds that $\nabla(\mathrm{O} / \mathrm{H})=$ $-0.026 \mathrm{dex} \mathrm{kpc}^{-1}$. From the estimate of $M_{\text {dark }} / M_{\text {tot }}$ in the solar vicinity by Bahcall, Flynn \& Gould (1992), it is obtained that $\nabla(\mathrm{O} / \mathrm{H}) \sim-0.03 \pm 0.01$ dex $\mathrm{kpc}^{-1}$; alternatively, from the $M_{\text {dark }} / M_{\text {tot }}$ estimate by Kroupa, Tout \& Gilmore (1993), it is found that $\nabla(\mathrm{O} / \mathrm{H})<-0.01 \mathrm{dex} \mathrm{kpc}^{-1}$; for these two estimates, we have assumed that: (a) $M_{\text {dark }} / M_{\text {tot }}$ increases with $R$ from a nul value at the galactic center, and (b) that $M_{\text {dark }}$ does not participate in the galactic chemical evolution process.

The outflow rate over the SFR is probably unimportant in the solar vicinity. Based on an analytical chemical evolution model of the solar vicinity and under the extreme assumption that there is no infall in the central regions of the Galaxy, Pagel (1989) finds that $\nabla(\mathrm{O} / \mathrm{H}) \sim-0.035 \mathrm{dex} \mathrm{kpc}-1$. From models of radial inflow, Köppen (1994) finds that it is possible to de- 
rive values as high as $-0.09 \mathrm{dex}^{\mathrm{kpc}}{ }^{-1}$ depending on two parameters: (a) the proportionality between the SFR and the gas density, and $(b)$ the radial flow velocities. Other gas flow possibilities are mentioned by Pagel (1989, 1992a, 1994).

The difference between closed models considering points (i) and (iii) above, and the observed gradients will help to constrain the gas flow effects considered in points (vii) to $(x)$.

\section{Planetary nebulae and abundance gradients}

Extragalactic PN have two advantages over galactic PN: (a) their galactocentric distances are better known, and $(b)$ their reddenings are, in general, smaller. Thay also have two advantages over extragalactic $\mathrm{H}$ II regions: (a) there are many more PN at all galactocentric distances, and (b) the wider range in ionization degrees allows better abundance determinations for some elements. Moreover, they provide us with qualitatively different information than H II regions, i. e., they trace the past abundance gradients, when their parent stars were formed, while H II regions trace the present abundance gradient.

It has been possible to derive chemical abundances of $\mathrm{PN}$ in galaxies of the local group. With the advent of the new generation of telescopes, it will be possible to derive the chemical composition of many more $\mathrm{PN}$ in galaxies of the local group and to extend this type of research to galaxies beyond the local group. The $\mathrm{O} / \mathrm{H}$ gradients derived from $\mathrm{PN}$ and $\mathrm{H}$ II regions might help to decide among the different models proposed by Köppen (1994) and Maciel \& Köppen (1994) and to assess the importance of infall in general.

$P N$ can be used to determine the S/O gradient in other galaxies, like M31, M33, and M81. These determinations might help to solve the S/O versus O/H controversy (e. g. Vilchez et al., 1988; Garnett 1989, 1992; Díaz et al., 1991; Pagel, 1992b; Henry \& Howard, 1994). While Vílchez et al. and Díaz et al. find that $\mathrm{S} / \mathrm{O}$ decreases with $\mathrm{O} / \mathrm{H}$ in nearby spiral galaxies, Garnett finds that $\mathrm{S} / \mathrm{O}$ is independent of $\mathrm{O} / \mathrm{H}$. The $\mathrm{S} / \mathrm{O}$ ratio is a function of the electron temperature, $T_{e}$, because the $S$ lines depend less on $T_{e}$ than the $O$ lines: therefore the $S / O$ discrepancy could be due to the $T_{e}$ calibration and consequently, the PN S/O determinations could help to check and possibly to improve the $([\mathrm{O}$ II $]+[\mathrm{O}$ III $]) / \mathrm{H}_{\beta}$ versus $\mathrm{O} / \mathrm{H}$ calibration used to derive the oxygen gradients.

\section{References}

Bahcall, J. N., Flynn, C. \& Gould, A. (1992) ApJ, 389, p. 234. Carigi, L. (1994) ApJ, 424, p. 181.

Carigi, L., Colín, P., Peimbert, M. \& Sarmiento, A. (1994) ApJ, submitted. 
Díaz, A. I., Terlevich, E., Vilchez, J. M., Pagel, B. E. J. \& Edmunds, M.

G. (1991) MNRAS, 253, p. 245.

Garnett, D. R. (1989) ApJ, 345, p. 282.

Garnett, D. R. (1992) $A J$, 103, p. 1330.

Güsten, R. \& Mezger, P. G. (1983) Vistas in Astr., 26, p. 159.

Henry, R. B. C. \& Howard, J. W. (1994) ApJ, in press.

Henry, R. B. C., Pagel, B. E. J., Lassiter, D. F. \& Chincarini, G. L. (1992) $M N R A S, \mathbf{2 5 8}$, p. 321.

Köppen, J. (1994) $A \cup B A, \mathbf{2 8 1}$, p. 26.

Kroupa, P., Tout, C. A. \& Gilmore, G. (1993) MNRAS, 262, p. 545.

Larson, R. B. (1986) MNRAS, 218, p. 409.

Maciel, W. J. \& Köppen, J. (1994) $A \mathcal{E} A$, 282, p. 436.

Maeder, A. (1992) $A \mathscr{E} A, \mathbf{2 6 4}$, p. 105.

McGaugh, S. S. (1991) ApJ, 380, p. 140.

Pagel, B. E. J. (1989) Rev. Mexicana Astron. Astrof., 18, p. 161.

Pagel, B. E. J. (1992a) in The Stellar Populations of Galaxies, IAU Symp. 149, eds. B. Barbuy \& A. Renzini (Kluwer Academic Publishers, Dordrecht), p. 133.

Pagel, B. E. J. (1992b) in The Feedback of Chemical Evolution on the Stellar Content of Galaxies, eds. D. Alloin \& G. Stasinska (París Observatoire), p. 87.

Pagel, B. E. J. (1994) in Galaxy Formation Ė Evolution, ed. C. MuñozTurón (Cambridge Univ. Press), in press.

Peimbert, M., Sarmiento. A. \& Colín, P. (1994) Rev. Mexicana Astron. Astrof., 28, p.181.

Peimbert, M. \& Serrano, A. (1982) MNRAS, 198, p. 563.

Richer, H. B., Fahlman, G. G., Buonanno, R., Fusi Pecci, F. , Searle, L. \& Thompson, I. B. (1991), ApJ. 381, p. 147.

Searle, L. \& Sargent, W. L. W. (1972) ApJ, 173, p. 25.

Vila-Costas, M. B. \& Edmunds, M. G. (1992) MNRAS, 259, p. 121.

Vilchez, J. M., Pagel, B. E. J., Díaz, A. I., Terlevich, E. \& Edmunds, M. G. (1988) MNRAS, 235, p. 633.

Zaritsky, D., Kennicutt. R. C. \& Huchra, J. P. (1994) ApJ, 420, p. 87. 